\title{
STABILITY AND STABILIZATION OF CONTINUOUS DESCRIPTOR SYSTEMS: AN LMI APPROACH
}

\author{
M. CHAABANE, O. BACHELIER, M. SOUISSI, AND D. MEHDI \\ Received 8 February 2005; Revised 19 November 2005; Accepted 24 January 2006
}

This paper deals with the problems of robust stability and stabilization for uncertain continuous descriptor systems. We propose a new necessary and sufficient condition in terms of a strict linear matrix inequality (LMI) for a nominal continuous descriptor system to be admissible (stable, regular, and impulse-free). Based on this, the state-feedback admissibility problem is solved and the solution is extended to the case of uncertain descriptor systems. Finally, numerical examples are given to illustrate the results.

Copyright (c) 2006 M. Chaabane et al. This is an open access article distributed under the Creative Commons Attribution License, which permits unrestricted use, distribution, and reproduction in any medium, provided the original work is properly cited.

\section{Introduction}

Intuitively, singular state-space description of linear systems is more general than conventional state-space description. In particular, a descriptor form includes information about static as well as dynamic constraints. Singular systems, both continuous and discrete, have been of interest in the literature since they have many applications (see [5]), for instance in electrical circuits network, robotics, and economics. It is fair to say that descriptor models give a more complete class of dynamical models than the conventional state-space systems.

Many classical concepts and results obtained for conventional systems have been extended to descriptor systems. Let us quote for instance controllability and observability, pole assignment, stability analysis $[8,10]$, and stabilization techniques as well as results including robustness aspects $[9,12,17]$.

The natural generalized Lyapunov equation (GLE) [10] was proven in [8] to fail unless the system is in its Weirstrass form and they proposed a new GLE equivalent to that given in [14]. In [12], the authors modified the GLE from [14] and proposed a matrix inequality equivalent condition.

In the available literature on descriptor systems, there are two kinds of stabilization problems for singular continuous-time systems. One consists in designing a state-feedback controller in such a way that the closed-loop system is regular, impulse-free, and 
stable or equivalently admissible. The other is to design a state-feedback controller in order to make the closed-loop system regular and stable.

In a number of approaches, the system model is transformed into a special form and it is understandable that this way of doing is not assuming very appropriate in the presence of uncertainty.

Concerning the stability analysis and the stabilization problem, a number of approaches assuming or not assuming the regularity of the descriptor system have been proposed in the literature let us quote for instance $[3,5,15]$ among those assuming the regularity and $[5,15]$ without assuming the regularity.

Robust control of linear state-space systems has been the focus of much attention during the past decades and various aspects and approaches for analysis and control design for linear uncertain systems have been investigated, see, for instance, [20]. In the available literature, we easily note that quadratic stability and stabilization approaches have taken a lion's share. The quadratic stability or stabilization is characterized by a determination of a unique so-called Lyapunov matrix which gives the approach an inherent conservatism. Many results have been reported in quadratic stability analysis and/or stabilization, see, for instance, $[2,19,20]$ and the references therein.

Recently the parameter dependent Lyapunov (PDL) approach has been introduced to reduce the conservatism of the quadratic approach. The PDL approach consists in expressing the Lyapunov matrix as a function of the uncertainty, and with the help of some slack additional variables, the approach yields a significant reduction of conservatism $[1,4,6]$.

In this paper, a linear matrix inequality (LMI) formulation is adopted to express necessary and/or sufficient conditions for the admissibility of continuous-time descriptor systems. The proposed approach can be understood as the LMI-correspondent formulation of the proposed GLE in [8]. It is known that strict inequality conditions are tractable and reliable especially with the available LMI software solver. Note that the conditions in [8] includes a nonstrict inequality associated with a standard Lyapunov inequality with the restriction that the solution is no longer symmetric and must satisfy an equality condition (see Remark 3.2). The admissibility property includes the stability as well as the regularity and the absence of impulses.

The state-feedback stabilization problem is solved by means of the admissibility of the closed loop. When the system contains uncertainties, the present method is extended to solve the robust state-feedback admissibility problem particularly through a PDL approach.

This paper is organized as follows. Section 2 gives the problem formulation and some preliminary definitions. Section 3 gives the result on admissibility for continuous-time descriptor systems. In Section 4, main result to solve the static feedback problem for the nominal descriptor systems is given, whereas Section 5 presents the result for uncertain singular systems. Section 6 presents illustrative examples. Section 7 concludes the paper.

\section{Problem formulation}

Consider the following continuous-time descriptor system:

$$
E \dot{x}(t)=A x(t)+B u(t)
$$


where $x(t) \in \mathbb{R}^{n}$ is the state, $u(t) \in \mathbb{R}^{m}$ is the control input. The matrix $E$ may be singular, we will assume that $\operatorname{rank} E=r \leq n$. $A$ and $B$ are known real constant matrices with appropriate dimensions.

Definition 2.1 [5]. (1) The pair $(E, A)$ is said to be regular if $\operatorname{det}(s E-A)$ is not identically zero.

(2) The pair $(E, A)$ is said to be impulse-free if $\operatorname{deg}(\operatorname{det}(s E-A))=\operatorname{rank} E$.

In the rest of the paper, the notation is standard unless it is otherwise specified. $L>0$ $(L<0)$ means that the matrix $L$ is a symmetric and positive definite matrix (a symmetric and negative definite). In the sequel, $\operatorname{Sym}\{\cdot\}$ is defined as $\operatorname{Sym}\{X\}=\left(X+X^{\top}\right)$ for any matrix $X$.

It is worth noting that the stability property for conventional systems is no more sufficient for singular systems but completed by the regularity and the absence of impulses and this lead us to introduce the notion of admissibility.

Definition $2.2[5,9]$. The continuous-time singular system (2.1) is said to be admissible if it is regular, impulse-free, and stable.

In order to characterize the admissibility of a singular system, let us recall that for a pair $(E, A)$, there exists a transformation couple $(U, V)$ such that

$$
\bar{E}=U E V=\left[\begin{array}{ll}
I & 0 \\
0 & 0
\end{array}\right], \quad \bar{A}=U A V=\left[\begin{array}{ll}
\bar{A}_{11} & \bar{A}_{12} \\
\bar{A}_{21} & \bar{A}_{22}
\end{array}\right] .
$$

It comes then that the singular system is said to be admissible if there exist a symmetric and positive matrix $\bar{X}_{11}$ and a nonsingular matrix $\bar{Y}_{22}$ such that the conditions

$$
\begin{gathered}
\operatorname{Sym}\left\{\left(\left(\bar{A}_{11}-\bar{A}_{12} \bar{A}_{22}^{-1} \bar{A}_{21}\right) \bar{X}_{11}\right)\right\}<0, \\
\operatorname{Sym}\left\{\bar{A}_{22} \bar{Y}_{22}\right\}<0
\end{gathered}
$$

hold.

Indeed, condition (2.4) means that the matrix $\bar{A}_{22}$ is nonsingular, whereas condition (2.3) states that the matrix $\left(\bar{A}_{11}-\bar{A}_{12} \bar{A}_{22}^{-1} \bar{A}_{21}\right)$ is stable. Notice that conditions (2.3) $-(2.4)$ are not tractable for uncertain system and it is preferable to use directly the system matrices. Both conditions will be combined in a unique condition as in [8]. The associated Lyapunov matrix will be neither symmetric nor positive definite. The positivity will be required only on a fraction of the Lyapunov matrix in order to satisfy condition (2.3).

To solve the admissibility problem, we propose a Lyaponuv-type admissibility condition, which is expressed by a strict LMI as given in Theorem 3.1. The goal of this paper is to find a static state-feedback controller $u(t)=K x(t)$ such that the closed-loop system $(E, A+B K)$ is admissible. This is defined as the static feedback admissibility problem of descriptor systems in this paper. The solvability of the above problems will be characterized by some LMI conditions. If the derived LMI conditions are feasible, the feedback gain matrix can be obtained. If the system contains polytopic uncertainties, the results can be modified to find the static state-feedback gain in such a way that the closed-loop uncertain system is admissible. 
4 Descriptor systems: stability and stabilization

\section{Stability analysis}

Consider the singular system described by the pair $(E, A)$ and define $E^{\perp}$ and $E^{\dagger}$ as follows:

$$
E^{\perp}=V(I-U E V) U, \quad E^{\dagger}=U^{-1}(I-U E V) U
$$

with $U$ and $V$ two nonsingular matrices satisfying

$$
U E V=\left[\begin{array}{ll}
I & 0 \\
0 & 0
\end{array}\right] .
$$

Theorem 3.1. The continuous-time singular system $(E, A)$ is admissible if and only if there exist some matrices $X, Y$, and $Z$ such that the matrix

$$
E X E^{\top}+\operatorname{Sym}\left\{E^{\dagger} Z\right\}
$$

is positive definite and

$$
M=\operatorname{Sym}\left\{\left(A X E^{\top}\right)\right\}+\operatorname{Sym}\left\{\left(A E^{\perp} Y\right)\right\}<0 .
$$

Remark 3.2. Note that in [12] the Lyapunov inequality has a solution which is no longer symmetric and must satisfy the condition $\left(V^{-1} P U^{\top}\right)_{12}=0$ and this comes from the equality constraint $E P=P E^{\top} \geq 0$. In Theorem 3.1 we have two strict LMI conditions, and (3.4) is in fact similar to the condition in [12] when we put $P=X E^{\top}+E^{\perp} Y$ and we get $E P=E X E^{\top}$ which is symmetric, and the positivity condition in $E P=P E^{\top} \geq 0$ is insured by (3.3) as a strict condition. Note also that a software as Matlab can not deal directly with nonstrict condition nor equality constraints.

Proof of Theorem 3.1

Sufficiency. First transform $M$ as follows:

$$
\begin{aligned}
\bar{M} & =U M U^{\top} \\
& =\operatorname{Sym}\left\{U A X E^{\top} U^{\top}\right\}+\operatorname{Sym}\left\{U A E^{\perp} Y U^{\top}\right\} \\
& =\operatorname{Sym}\left\{\overline{A X E}^{\top}\right\}+\operatorname{Sym}\left\{\overline{A E}^{\perp} \bar{Y}\right\},
\end{aligned}
$$

with

$$
\begin{aligned}
\bar{A}=U A V= & {\left[\begin{array}{ll}
\bar{A}_{11} & \bar{A}_{12} \\
\bar{A}_{21} & \bar{A}_{22}
\end{array}\right], \quad \bar{E}=U E V=\left[\begin{array}{ll}
I & 0 \\
0 & 0
\end{array}\right], \quad \bar{X}=V^{-1} X V^{-\top}=\left[\begin{array}{ll}
\bar{X}_{11} & \bar{X}_{12} \\
\bar{X}_{21} & \bar{X}_{22}
\end{array}\right], } \\
& \bar{E}^{\perp}=V^{-1} E^{\perp} U^{-1}=\left[\begin{array}{ll}
0 & 0 \\
0 & I
\end{array}\right], \quad \bar{Y}=U Y U^{\top}=\left[\begin{array}{ll}
\bar{Y}_{11} & \bar{Y}_{12} \\
\bar{Y}_{21} & \bar{Y}_{22}
\end{array}\right] .
\end{aligned}
$$


It comes then that

$$
\bar{M}=\operatorname{Sym}\left\{\left[\begin{array}{ll}
\left(\bar{A}_{11} \bar{X}_{11}+\bar{A}_{12} \bar{X}_{21}\right) & 0 \\
\left(\bar{A}_{21} \bar{X}_{11}+\bar{A}_{22} \bar{X}_{21}\right) & 0
\end{array}\right]\right\}+\operatorname{Sym}\left\{\left[\begin{array}{ll}
\left(\bar{A}_{12} \bar{Y}_{21}\right) & \left(\bar{A}_{12} \bar{Y}_{22}\right) \\
\left(\bar{A}_{22} \bar{Y}_{21}\right) & \left(\bar{A}_{22} \bar{Y}_{22}\right)
\end{array}\right]\right\}
$$

which shows that $\bar{M}<0$ necessarily implies

$$
\operatorname{Sym}\left\{\bar{A}_{22} \bar{Y}_{22}\right\}<0 \text {, }
$$

and this means that $\bar{A}_{22}$ is invertible or in other words that the system is regular and impulse-free.

Moreover, consider the two matrices

$$
\Sigma=\left[\begin{array}{cc}
I & 0 \\
-\bar{A}_{22}^{-1} \bar{A}_{21} & \bar{A}_{22}
\end{array}\right], \quad \Gamma=\left[\begin{array}{cc}
I & -\bar{A}_{12} \bar{A}_{22}^{-1} \\
0 & I
\end{array}\right]
$$

that transform the matrix $\bar{A}$ in a diagonal form as

$$
\overline{\bar{A}}=\Gamma \bar{A} \Sigma=\left[\begin{array}{cc}
\overline{\bar{A}}_{11} & 0 \\
0 & I
\end{array}\right]
$$

with

$$
\overline{\bar{A}}_{11}=\bar{A}_{11}-\bar{A}_{12} \bar{A}_{22}^{-1} \bar{A}_{21}
$$

and transform $\bar{M}$ into $\overline{\bar{M}}$ as

$$
\overline{\bar{M}}=\Gamma \overline{\bar{M}} \Gamma^{\top}=\operatorname{Sym}\left\{\overline{\overline{A X E}}^{\top}\right\}+\operatorname{Sym}\left\{\overline{\overline{A E}}^{\perp} \overline{\bar{Y}}\right\}
$$

with

$$
\begin{aligned}
\overline{\bar{E}} & =\Gamma \bar{E} \Sigma=\bar{E}, \\
\overline{\bar{X}} & =\Sigma^{-1} \bar{X} \Sigma^{\top-1}=\left[\begin{array}{cc}
\bar{X}_{11} & * \\
* & *
\end{array}\right], \\
\overline{\bar{E}}^{\perp} & =\Sigma^{-1} \bar{E}^{\perp} \Gamma^{-1}=\bar{E}^{\perp}, \\
\overline{\bar{Y}} & =\Gamma \bar{Y} \Gamma^{\top} .
\end{aligned}
$$

It comes then that

$$
\overline{\bar{M}}=\operatorname{Sym}\left\{\left[\begin{array}{cc}
\overline{\bar{A}}_{11} \bar{X}_{11} & 0 \\
* & 0
\end{array}\right]\right\}+\operatorname{Sym}\left\{\left[\begin{array}{ll}
0 & 0 \\
* & *
\end{array}\right]\right\}
$$


where the $*$ corresponds to entries with no much relevance at this step of arguments. Note that if $\overline{\bar{M}}$ is negative definite, then necessarily we have

$$
\operatorname{Sym}\left\{\overline{\bar{A}}_{11} \bar{X}_{11}\right\}<0 \text {. }
$$

Condition (3.15) means that the singular system is stable if matrix $\bar{X}_{11}$ is symmetric and positive definite and this comes from condition (3.3) as follows: notice that

$$
\begin{aligned}
U\left(E X E^{\top}+\operatorname{Sym}\left\{E^{\dagger} Z\right\}\right) U^{\top} & =U E V V^{-1} X V^{-\top}(U E V)^{\top}+\operatorname{Sym}\left\{U E^{\dagger} U^{-1} U Z U^{\top}\right\} \\
& =\overline{E X E}+\operatorname{Sym}\left\{\bar{E}^{\dagger} \bar{Z}\right\} \\
& =\left[\begin{array}{ll}
I & 0 \\
0 & 0
\end{array}\right]\left[\begin{array}{cc}
\bar{X}_{11} & * \\
* & *
\end{array}\right]\left[\begin{array}{ll}
I & 0 \\
0 & 0
\end{array}\right]+\operatorname{Sym}\left\{\left[\begin{array}{ll}
0 & 0 \\
0 & I
\end{array}\right]\left[\begin{array}{cc}
* & * \\
* & \bar{Z}_{22}
\end{array}\right]\right\},
\end{aligned}
$$

which means that condition (3.3) is equivalent to

$$
\overline{\bar{X}}_{11}>0
$$

and this ends the proof of the sufficiency part.

Necessity. Assume that the system is stable, regular, and impulse-free or in other words there exist two matrices $\overline{\bar{X}}_{11}>0$ and $\overline{\bar{Y}}_{22}$ such that

$$
\begin{aligned}
& \operatorname{Sym}\left\{\left(\overline{\bar{A}}_{11} \overline{\bar{X}}_{11}\right)\right\}<0, \\
& \operatorname{Sym}\left\{\overline{\bar{A}}_{22} \overline{\bar{Y}}_{22}\right\}<0,
\end{aligned}
$$

then one can surely find two matrices $\overline{\bar{X}}_{21}$ and $\overline{\bar{Y}}_{21}$ in such a way that the LMI

$$
\operatorname{Sym}\left\{\left[\begin{array}{ll}
\left(\overline{\bar{A}}_{11} \overline{\bar{X}}_{11}\right) & 0 \\
\left(\overline{\bar{A}}_{22} \overline{\bar{X}}_{21}\right) & 0
\end{array}\right]\right\}+\operatorname{Sym}\left\{\left[\begin{array}{cc}
0 & 0 \\
\left(\overline{\bar{A}}_{22} \overline{\bar{Y}}_{21}\right) & \left(\overline{\bar{A}}_{22} \overline{\bar{Y}}_{22}\right)
\end{array}\right]\right\}<0
$$

is satisfied. Indeed, if $\overline{\bar{X}}_{21}$ and $\overline{\bar{Y}}_{21}$ satisfy the condition

$$
\operatorname{Sym}\left\{\left(\overline{\bar{A}}_{11} \overline{\bar{X}}_{11}\right)\right\}-\left(\overline{\bar{X}}_{21}+\overline{\bar{Y}}_{21}\right)^{\top} \overline{\bar{A}}_{22}^{\top}\left(\operatorname{Sym}\left\{\overline{\bar{A}}_{22} \overline{\bar{Y}}_{22}\right\}\right)^{-1} \overline{\bar{A}}_{22}\left(\overline{\bar{X}}_{21}+\overline{\bar{Y}}_{21}\right)<0,
$$

then (3.19) holds. Note that condition (3.19) is in fact

$$
\operatorname{Sym}\left\{\left(\overline{\overline{A X E}}^{\top}\right)\right\}+\operatorname{Sym}\left\{\left(\overline{\overline{A E}}^{\perp} \overline{\bar{Y}}\right)\right\}<0 .
$$

Then with similar arguments as in the sufficiency part one can recover condition (3.3) which closes the proof of the theorem. 


\section{Stabilization by state feedback}

In this section, we address the problem of stabilization by state feedback for the singular system given by

$$
E \dot{x}(t)=A x(t)+B u(t)
$$

where $x(t) \in \mathbb{R}^{n}$ is the state and $u(t) \in \mathbb{R}^{m}$ is the control input.

The control law given by a state feedback is then

$$
u(t)=K x(t),
$$

where the gain $K$ of appropriate dimension is computed in such a way that the singular closed-loop system is admissible.

THeORem 4.1. If the LMI problem

$$
\begin{gathered}
E X E^{\top}+\operatorname{Sym}\left\{E^{\dagger} Z\right\}>0, \\
\operatorname{Sym}\left\{\left(X E^{\top}+E^{\perp} Y\right)\right\}>0, \\
{\left[\begin{array}{cc}
0 & \left(X E^{\top}+E^{\perp} Y\right)^{\top} \\
\left(X E^{\top}+E^{\perp} Y\right) & 0
\end{array}\right]+\operatorname{Sym}\left\{\left[\begin{array}{c}
A G+B R \\
-G
\end{array}\right]\left[\begin{array}{ll}
I & I
\end{array}\right]\right\}<0}
\end{gathered}
$$

is feasible in the variables $X, Y, Z, R$, and $G$, then the closed-loop singular system is admissible with the state feedback

$$
K=R G^{-1}
$$

Remark 4.2. It is fair to mention that the result in [7] combined with the criterion in [2] leads also to a condition where the Lyapunov matrix is decoupled.

Proof of Theorem 4.1. The closed-loop singular system is admissible if (and only if) there exist two matrices $X$ and $Y$ and a state feedback $K$ with matrix $X$ satisfying condition (4.3) and

$$
\operatorname{Sym}\left\{(A+B K)\left(X E^{\top}+E^{\perp} Y\right)\right\}<0 .
$$

The above equation can be written as

$$
\left[\begin{array}{ll}
I & A+B K
\end{array}\right]\left[\begin{array}{cc}
0 & \left(X E^{\top}+E^{\perp} Y\right)^{\top} \\
\left(X E^{\top}+E^{\perp} Y\right) & 0
\end{array}\right]\left[\begin{array}{c}
I \\
(A+B K)^{\top}
\end{array}\right]<0,
$$

and in the same way we note that (4.4) can be rewritten as

$$
\operatorname{Sym}\left\{-\left(X E^{\top}+E^{\perp} Y\right)\right\}=\left[\begin{array}{ll}
I & -I
\end{array}\right]\left[\begin{array}{cc}
0 & \left(X E^{\top}+E^{\perp} Y\right)^{\top} \\
\left(X E^{\top}+E^{\perp} Y\right) & 0
\end{array}\right]\left[\begin{array}{c}
I \\
-I
\end{array}\right]<0 .
$$


Thus, according to the elimination lemma [13, Theorem 2.3.12], conditions (4.8) and (4.9) hold if and only if there exists a matrix $G$ satisfying the condition

$$
\left[\begin{array}{cc}
0 & \left(X E^{\top}+E^{\perp} Y\right)^{\top} \\
\left(X E^{\top}+E^{\perp} Y\right) & 0
\end{array}\right]+\operatorname{Sym}\left\{\left[\begin{array}{c}
(A+B K) G \\
-G
\end{array}\right]\left[\begin{array}{ll}
I & I
\end{array}\right]\right\}<0,
$$

and it becomes clear that a change of variable $R=K G$ yields condition (4.5).

It is worth noting that condition (4.4) has been added in Theorem 4.1 in order to obtain condition (4.5) which has the consequence to eliminate the necessity of the condition.

From Theorem 4.1, we can easily be aware that this approach has the advantage to be nicely applicable in the case the system is uncertain with a polytopic description of uncertainty which is the subject of the next section.

Remark 4.3. Theorem 4.1 can easily be extended to system with decentralized control described by the model [18]

$$
E \dot{x}(t)=A x(t)+\sum_{i}^{N} B_{i} u_{i}(t)
$$

with the control law

$$
u_{i}(t)=K_{i} x(t)
$$

In Theorem 4.1, only condition (4.5) has to be replaced by

$$
\left[\begin{array}{cc}
0 & \left(X E^{\top}+E^{\perp} Y\right)^{\top} \\
\left(X E^{\top}+E^{\perp} Y\right) & 0
\end{array}\right]+\operatorname{Sym}\left\{\left[\begin{array}{c}
A G+\mathscr{B} \mathscr{R} \\
-G
\end{array}\right]\left[\begin{array}{ll}
I & I
\end{array}\right]\right\}<0
$$

with

$$
\mathscr{B}=\left[\begin{array}{llll}
B_{1} & B_{2} & \cdots & B_{N}
\end{array}\right], \quad \mathscr{R}=\left[\begin{array}{llll}
R_{1}^{\top} & R_{2}^{\top} & \cdots & R_{N}^{\top}
\end{array}\right]^{\top},
$$

and the controller will be given by

$$
K_{i}=R_{i} G^{-1}, \quad i=1, \ldots, N .
$$

\section{Robust stabilization}

In this section, we consider the feedback stabilization problem for systems containing uncertainties. The descriptor system is characterized by the pair $(E, A(\bar{\alpha}, \Delta), B(\bar{\alpha}, \Delta))$, where matrices $A(\bar{\alpha}, \Delta)$ and $B(\bar{\alpha}, \Delta)$ are given as

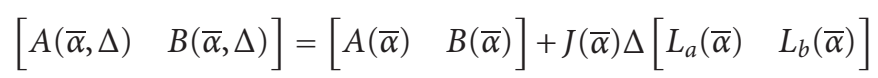

with matrix $\Delta$ representing the norm-bounded uncertainty satisfying the constraint

$$
\Delta^{\top} \Delta<\rho^{2} I
$$


and $\rho$ corresponds to the norm-bounded uncertainty radius which, in fact, has to be maximized.

Matrices $A(\bar{\alpha}), B(\bar{\alpha}), J(\bar{\alpha}), L_{a}(\bar{\alpha})$, and $L_{b}(\bar{\alpha})$ belong to a polytope, that is

$$
\left[\begin{array}{lllll}
A(\bar{\alpha}) & B(\bar{\alpha}) & J(\bar{\alpha}) & L_{a}(\bar{\alpha}) & L_{b}(\bar{\alpha})
\end{array}\right]=\sum_{i=1}^{p} \alpha_{i}\left[\begin{array}{lllll}
A_{i} & B_{i} & J_{i} & L_{a_{i}} & L_{b_{i}}
\end{array}\right]
$$

with

$$
\begin{gathered}
\alpha_{i} \geq 0, \quad i=1, \ldots, p \\
\sum_{i=1}^{p} \alpha_{i}=1, \quad \bar{\alpha}=\left[\begin{array}{lll}
\alpha_{1} & \ldots & \alpha_{p}
\end{array}\right] .
\end{gathered}
$$

The problem for a system corrupted by uncertainty is to preserve its performances for all admissible uncertainties or in other terms for every instance of matrices $A(\bar{\alpha}, \Delta)$ and $B(\bar{\alpha}, \Delta)$.

Definition 5.1. The uncertain singular system is robustly admissible if the eigenvalues of the pencil matrix $(E, A(\bar{\alpha}), \Delta)$ lie in the open left half-plane for all admissible uncertainty $\bar{\alpha}$ and $\Delta$.

Definition 5.2. The uncertain singular system is said to be quadratically admissible if there exist a symmetric and positive definite matrix $\bar{X}_{11}$ and a nonsingular matrix $\bar{Y}_{22}$ such that

$$
\begin{gathered}
\operatorname{Sym}\left\{\left(\left(\bar{A}_{11}(\bar{\alpha}, \Delta)-\bar{A}_{12}(\bar{\alpha}, \Delta) \bar{A}_{22}^{-1}(\bar{\alpha}, \Delta) \bar{A}_{21}(\bar{\alpha}, \Delta)\right) \bar{X}_{11}\right)\right\}<0, \\
\operatorname{Sym}\left\{\bar{A}_{22}(\bar{\alpha}, \Delta) \bar{Y}_{22}\right\}<0
\end{gathered}
$$

hold.

Recall that quadratic stability implies robust stability but the converse is, in general, false. This comes from the fact that for quadratic stability there exists a single couple of matrices $\left(\bar{X}_{11}, \bar{Y}_{22}\right)$ for all admissible uncertainties. If quadratic admissibility is only sufficient for robust admissibility, it matters to find (sufficient) conditions for robust admissibility that are less conservative than quadratic admissibility. In other words, it is important to derive conditions implicitly involving matrices $X$ and $Y$ that are not constant but dependent on the uncertainty. One possibility is that matrices $X$ and $Y$ comply with

$$
\left[\begin{array}{ll}
X & Y
\end{array}\right]=\sum_{i=1}^{p} \alpha_{i}\left[\begin{array}{ll}
X_{i} & Y_{i}
\end{array}\right]
$$

where $X_{i}$ and $Y_{i}$ are valid to assess the admissibility of extreme models. Based upon these notions, the next theorem for $\Delta=0$, that is without the norm-bounded uncertainty, is proposed. 
THEOREM 5.3. The uncertain singular system is robustly admissible if there exist matrices $X_{i}, Y_{i}, v Z_{i}$, for $i=1, \ldots, p$, and two matrices $G$ and $R$ such that the LMI problem

$$
\begin{gathered}
E X_{i} E^{\top}+\operatorname{Sym}\left\{E^{\dagger} Z_{i}\right\}>0, \\
\operatorname{Sym}\left\{\left(X_{i} E^{\top}+E^{\perp} Y_{i}\right)\right\}>0 \\
{\left[\begin{array}{cc}
0 & \left(X_{i} E^{\top}+E^{\perp} Y_{i}\right)^{\top} \\
\left(X_{i} E^{\top}+E^{\perp} Y_{i}\right) & 0
\end{array}\right]+\operatorname{Sym}\left\{\left[\begin{array}{c}
A_{i} G+B_{i} R \\
-G
\end{array}\right]\left[\begin{array}{ll}
I & I
\end{array}\right]\right\}<0}
\end{gathered}
$$

is feasible in the variables $X_{i}, Y_{i}, V$, and $R$, then the admissible feedback gain is given by

$$
K=R G^{-1}
$$

Remark 5.4. In [11], the authors propose a proportional and derivative (PD) controller. This PD controller allows them to use an augmented system for which the corresponding matrix $E$ is in the form

$$
\left[\begin{array}{ll}
I & 0 \\
0 & 0
\end{array}\right]
$$

yielding a single LMI condition, whereas in the present work we restrict ourselves to a simple proportional controller.

Proof of Theorem 5.3. Let matrices $X$ and $Y$ be defined as in (5.6), then multiplying conditions (5.7) by $\alpha_{i}$ and summing up from 1 to $p$, one gets the same conditions as in Theorem 4.1.

In the case where we consider norm-bounded uncertainties, we need the following lemma [16].

Lemma 5.5. Let $Z, E, F$, and $\Delta$ be matrices of appropriate dimensions. Assume that $Z$ is symmetric, and $\Delta^{\top} \Delta \leq I$, then

$$
Z+E \Delta F+F^{\top} \Delta^{\top} E^{\top}<0
$$

if and only if there exists a scalar $\lambda>0$ satisfying

$$
Z+\lambda E E^{\top}+\lambda^{-1} F^{\top} F<0
$$

Lemma 5.5 enables us to propose the following theorem in the case of both uncertainties, that is, polytopic and norm bounded.

THeOREM 5.6. The uncertain singular system is robustly admissible if there exist matrices $X_{i}, Y_{i}, Z_{i}$, for $i=1, \ldots, p$, and two matrices $G$ and $R$ and $a$ positive scalar $\lambda$ such that the 
LMI problem

$$
\begin{aligned}
& E X_{i} E^{\top}+\operatorname{Sym}\left\{E^{\dagger} Z_{i}\right\}>0, \\
& \operatorname{Sym}\left\{\left(X_{i} E^{\top}+E^{\perp} Y_{i}\right)\right\}>0, \\
& {\left[\begin{array}{cccc}
0 & \left(X_{i} E^{\top}+E^{\perp} Y_{i}\right)^{\top} & \left(L_{a_{i}} G+L_{b_{i}} R\right)^{\top} & \lambda \rho J_{i} \\
\left(X_{i} E^{\top}+E^{\perp} Y_{i}\right) & 0 & \left(L_{a_{i}} G+L_{b_{i}} R\right)^{\top} & 0 \\
L_{a_{i}} G+L_{b_{i}} R & L_{a_{i}} G+L_{b_{i}} R & -\lambda I & 0 \\
\lambda \rho J_{i}^{\top} & 0 & 0 & -\lambda I
\end{array}\right]} \\
& +\operatorname{Sym}\left\{\left[\begin{array}{c}
A_{i} G+B_{i} R \\
-G \\
0 \\
0
\end{array}\right]\left[\begin{array}{llll}
I & I & 0 & 0
\end{array}\right]\right\}<0
\end{aligned}
$$

is feasible in the variables $X_{i}, Y_{i}, G$, and $R$; then the admissible feedback gain is given by

$$
K=R G^{-1}
$$

Remark 5.7. Note that we can take $\lambda=1$ in condition (5.14) and in fact all the decision variables will comply to the following change of variable given here only for $X_{i}: \lambda^{-1} X_{i}$.

Proof of Theorem 5.6. The proof of the previous theorem is straightforward from Theorem 2.1 and Lemma 5.5. That is, in the case of both polytopic and norm-bounded uncertainties we take $X$ and $Y$ according to (5.6) and apply Theorem 4.1 where conditions (4.3) and (4.4) remain unchanged and condition (4.5) becomes

$$
\begin{aligned}
& \mathcal{N}(\alpha, \Delta)=\left[\begin{array}{cc}
0 & \left(X(\alpha) E^{\top}+E^{\perp} Y(\alpha)\right)^{\top} \\
\left(X(\alpha) E^{\top}+E^{\perp} Y(\alpha)\right) & 0
\end{array}\right] \\
& +\operatorname{Sym}\left\{\left[\begin{array}{c}
\left(A(\alpha)+J(\alpha) \Delta L_{a}(\alpha)\right) G+\left(B(\alpha)+J(\alpha) \Delta L_{b}(\alpha)\right) R \\
-G
\end{array}\right]\left[\begin{array}{ll}
I & I
\end{array}\right]\right\}<0 \\
& =\left[\begin{array}{cc}
0 & \left(X(\alpha) E^{\top}+E^{\perp} Y(\alpha)\right)^{\top} \\
\left(X(\alpha) E^{\top}+E^{\perp} Y(\alpha)\right) & 0
\end{array}\right] \\
& +\operatorname{Sym}\left\{\left[\begin{array}{c}
A(\alpha) G+B(\alpha) R \\
-G
\end{array}\right]\left[\begin{array}{ll}
I & I
\end{array}\right]\right\} \\
& +\operatorname{Sym}\left\{\left[\begin{array}{c}
J(\alpha) \\
0
\end{array}\right] \Delta\left[L_{a}(\alpha) G+L_{b}(\alpha) R \quad L_{a}(\alpha) G+L_{b}(\alpha) R\right]\right\}<0,
\end{aligned}
$$


and according to Lemma 5.5, this holds if there exists a positive scalar $\lambda$ such that we have

$$
\begin{aligned}
& {\left[\begin{array}{cccc}
0 & \left(X(\alpha) E^{\top}+E^{\perp} Y(\alpha)\right)^{\top} & \left(L_{a}(\alpha) G+L_{b}(\alpha) R\right)^{\top} & \lambda \rho J(\alpha) \\
\left(X(\alpha) E^{\top}+E^{\perp} Y(\alpha)\right) & 0 & \left(L_{a}(\alpha) G+L_{b}(\alpha) R\right)^{\top} & 0 \\
L_{a}(\alpha) G+L_{b}(\alpha) R & L_{a}(\alpha) G+L_{b}(\alpha) R & -\lambda I & 0 \\
\lambda \rho J^{\top}(\alpha) & 0 & 0 & -\lambda I
\end{array}\right]} \\
& +\operatorname{Sym}\left\{\left[\begin{array}{c}
A(\alpha) G+B(\alpha) R \\
-G \\
0 \\
0
\end{array}\right]\left[\begin{array}{llll}
I & I & 0 & 0
\end{array}\right]\right\}<0
\end{aligned}
$$

from which we get easily condition (5.14) and this ends the proof.

Remark 5.8. It is worth noting that we can also extend Remark 4.3 to the case of uncertain decentralized control systems with both polytopic and norm-bounded uncertainty.

\section{Illustrative example}

Example 6.1. Consider a continuous-time descriptor system as in (4.1) described by the parameters (see [9])

$$
E=\left[\begin{array}{ccc}
1 & 0 & 0 \\
0 & 1 & 1 \\
-1 & 1 & 1
\end{array}\right], \quad A=\left[\begin{array}{ccc}
0 & 1 & 0 \\
-1 & 3 & 3 \\
-1 & 2 & 3
\end{array}\right], \quad B=\left[\begin{array}{ll}
0.0 & 0.2 \\
1.0 & 0.0 \\
0.9 & 0.8
\end{array}\right]
$$

The finite poles are located at 0.3820 and 2.6180 which implies that the open loop is not admissible.

By Theorem 4.1 and using the Matlab LMI solver, we obtain

$$
\begin{aligned}
& X=\left[\begin{array}{ccc}
0.0024 & -0.3093 & 0.3100 \\
-0.3093 & -1.8084 & 1.1614 \\
0.3100 & 1.1614 & -0.5117
\end{array}\right] \times 10^{4}, \\
& Y=\left[\begin{array}{ccc}
-1.0360 & 1.9258 & 3.1217 \\
-2.0579 & -1.4776 & -0.1499 \\
2.0579 & 3.0883 & 0.1034
\end{array}\right] \times 10^{3}, \\
& G=\left[\begin{array}{ccc}
16.4377 & 88.2391 & 341.4507 \\
-92.8939 & 7.7904 & -261.7340 \\
-341.2044 & 263.9527 & 14.5597
\end{array}\right], \\
& R=\left[\begin{array}{ccc}
1.3960 & -0.7423 & 1.3427 \\
0.4106 & -0.3908 & -0.5017
\end{array}\right] \times 10^{3},
\end{aligned}
$$


and the state-feedback gain

$$
K=R G^{-1}=\left[\begin{array}{ccc}
-0.0293 & -5.3153 & -2.6456 \\
2.3622 & 4.8644 & -2.4139
\end{array}\right]
$$

which renders the resulting closed-loop system admissible with the finite closed-loop poles located at $-0.3517+0.1969 i$ and $-0.3517-0.1969 i$.

Example 6.2. Consider the uncertain singular system defined by

$$
E=\left[\begin{array}{lll}
1 & 0 & 0 \\
0 & 1 & 0 \\
0 & 0 & 0
\end{array}\right]
$$

with matrices $A$ and $B$ belonging to a polytope whose vertices are given by the state matrices

$$
\begin{aligned}
& {\left[\begin{array}{ll}
A_{1} & A_{2}
\end{array}\right]=\left[\begin{array}{cccccc}
0.7918 & 0.0792 & 0.1362 & 0.6318 & 0.0680 & 0.1162 \\
0.6900 & 0.6762 & 2.3232 & 0.7500 & 0.6162 & 2.3632 \\
0 & 0 & 0.6688 & 0 & 0 & 0.6088
\end{array}\right],} \\
& {\left[\begin{array}{ll}
A_{3} & A_{4}
\end{array}\right]=\left[\begin{array}{cccccc}
0.7918 & 0.0800 & 0.1362 & 0.6318 & 0.0672 & 0.1162 \\
0.8900 & 0.6762 & 2.5632 & 0.5500 & 0.6162 & 2.1232 \\
0 & 0 & 0.6688 & 0 & 0 & 0.6088
\end{array}\right] .}
\end{aligned}
$$

The input matrices

$$
\begin{aligned}
{\left[\begin{array}{lllll}
B_{1} & B_{2} & B_{3} & B_{4}
\end{array}\right] } \\
=\left[\begin{array}{llllllll}
0.0122 & 0.0422 & 0.0122 & 0.0402 & 0.0122 & 0.0422 & 0.0122 & 0.0402 \\
0.3468 & 0.1230 & 0.3628 & 0.1230 & 0.3868 & 0.1230 & 0.3228 & 0.1230 \\
0.1945 & 0.2141 & 0.2085 & 0.2461 & 0.2225 & 0.2541 & 0.1805 & 0.2061
\end{array}\right] .
\end{aligned}
$$

According to Theorem 5.3, the robust state-feedback gain

$$
K=\left[\begin{array}{ccc}
1.8265 & -6.6699 & -7.2433 \\
-11.7288 & 5.8273 & 2.1016
\end{array}\right]
$$

will robustly render the uncertain closed-loop system stable, regular, and impulse free.

\section{Conclusion}

The problem of stability and stabilization for continuous-time descriptor systems has been studied. In terms of a strict LMI, a necessary and sufficient condition for continuous 
descriptor systems to be admissible has been proposed. This condition is an LMI twin formulation of the well-known improved generalized Lyapunov equation. LMI conditions are obtained to ensure the admissibility of the closed loop via a state-feedback control law. A robust admissible state feedback control law is proposed for polytopic uncertain continuous descriptor systems. Numerical examples are given to illustrate the usefulness of the proposed methods.

\section{References}

[1] O. Bachelier, J. Bernussou, M.-C. de Oliveira, and J.-C. Geromel, Parameter dependent Lyapunov control design: numerical evaluation, Proceedings of the 38th IEEE Conference on Decision \& Control, Arizona, December 1999, pp. 293-297.

[2] B. R. Barmish, Necessary and sufficient conditions for quadratic stabilizability of an uncertain system, Journal of Optimization Theory and Applications 46 (1985), no. 4, 399-408.

[3] P. Bernhard, On singular implicit linear dynamical systems, SIAM Journal on Control and Optimization 20 (1982), no. 5, 612-633.

[4] J. Daafouz and J. Bernussou, Parameter dependent Lyapunov functions for discrete time systems with time varying parametric uncertainties, Systems \& Control Letters 43 (2001), no. 5, 355359.

[5] L. Dai, Singular Control Systems, Lecture Notes in Control and Information Sciences, vol. 118, Springer, Berlin, 1989.

[6] P. Gahinet, P. Apkarian, and M. Chilali, Affine parameter-dependent Lyapunov functions and real parametric uncertainty, IEEE Transactions on Automatic Control 41 (1996), no. 3, 436-442.

[7] J.-C. Geromel, M.-C. de Oliveira, and L. Hsu, LMI characterization of structural and robust stability, Linear Algebra and Its Applications 285 (1998), no. 1-3, 69-80.

[8] J. Y. Ishihara and M. H. Terra, On the Lyapunov theorem for singular systems, IEEE Transactions on Automatic Control 47 (2002), no. 11, 1926-1930.

[9] C.-H. Kuo and C.-H. Fang, An LMI approach to admissibilization of unceratin descriptor systems via static output feedback, Proceedings of the American Control Conference, vol. 6, Colorado, June 2003, pp. 5104-5109, FP06.

[10] F. L. Lewis, A survey of linear singular systems, Circuits, Systems, and Signal Processing 5 (1986), no. 1, 3-36.

[11] C. Lin, Q.-G. Wang, and T. H. Lee, Robust normalization and stabilization of uncertain descriptor systems with norm-bounded perturbations, IEEE Transactions on Automatic Control 50 (2005), no. 4, 515-520.

[12] I. Masubuchi, Y. Kamitane, A. Ohara, and N. Suda, $H_{\infty}$ control for descriptor systems: a matrix inequalities approach, Automatica 33 (1997), no. 4, 669-673.

[13] R. E. Skelton, T. Iwasaki, and K. M. Grigoriadis, A Unified Algebraic Approach to Linear Control Design, The Taylor \& Francis Systems and Control Book Series, Taylor \& Francis, London, 1998.

[14] K. Takaba, N. Morihira, and T. A. Katayama, A generalized Lyapunov theorem for descriptor system, Systems \& Control Letters 24 (1995), no. 1, 49-51.

[15] A. Varga, On stabilization methods of descriptor systems, Systems \& Control Letters 24 (1995), no. $2,133-138$.

[16] L. Xie, Output feedback $H_{\infty}$ control of systems with parameter uncertainty, International Journal of Control 63 (1996), no. 4, 741-750.

[17] S. Xu and J. Lam, Robust stability and stabilization of discrete singular systems: an equivalent characterization, IEEE Transactions on Automatic Control 49 (2004), no. 4, 568-574.

[18] R. Yu, Regularizability of linear time-invariant descriptor systems under decentralized control, Automatica 41 (2005), no. 9, 1639-1644. 
[19] K. Zhou, J.-C. Doyle, and K. Glover, Robust and Optimal Control, Prentice-Hall, New Jersey, 1996.

[20] K. Zhou and P. P. Khargonekar, Robust stabilization of linear systems with norm-bounded timevarying uncertainty, Systems \& Control Letters 10 (1988), no. 1, 17-20.

M. Chaabane: Automatic Control Unit, Sfax Preparatory Institute for Engineering Studies (IPEIS), Sfax University, BP 805, 3018 Sfax, Tunisia

E-mail address: chaabane_uca@yahoo.com

O. Bachelier: Laboratory of Automatic Control and Computer Science for Industry (LAII),

Higher Engineering School of Poitiers (ESIP), University of Poitiers, 40 Avenue du Recteur Pineau, 86022 Poitiers Cedex, France

E-mail address: olivier.bachelier@univ-poitiers.fr

M. Souissi: Automatic Control Unit, Sfax Preparatory Institute for Engineering Studies (IPEIS), Sfax University, BP 805, 3018 Sfax,Tunisia

E-mail address: mansour.souissi@ipeis.rnu.tn

D. Mehdi: Laboratory of Automatic Control and Computer Science for Industry (LAII),

Higher Engineering School of Poitiers (ESIP), University of Poitiers, 40 Avenue du Recteur Pineau, 86022 Poitiers Cedex, France

E-mail address: mehdi.driss@univ-poitiers.fr 


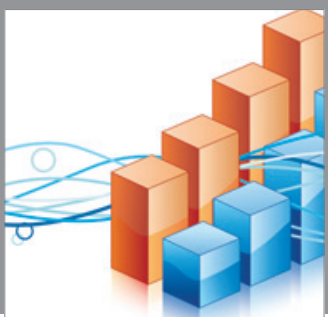

Advances in

Operations Research

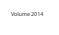

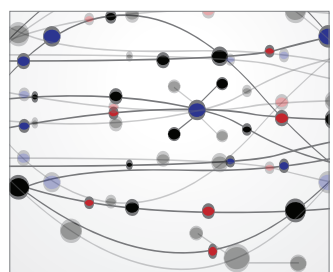

\section{The Scientific} World Journal
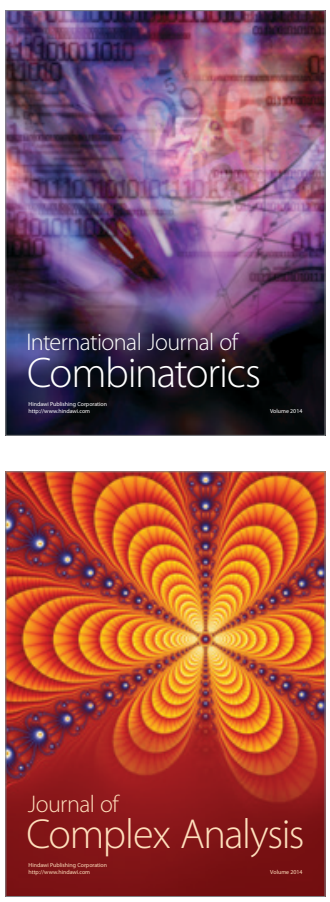

International Journal of

Mathematics and

Mathematical

Sciences
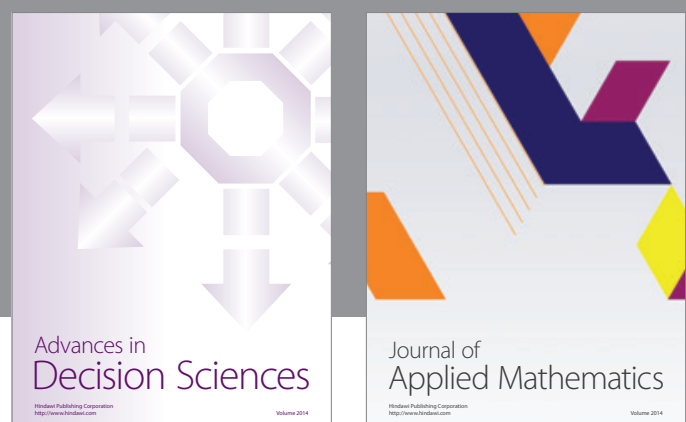

Journal of

Applied Mathematics
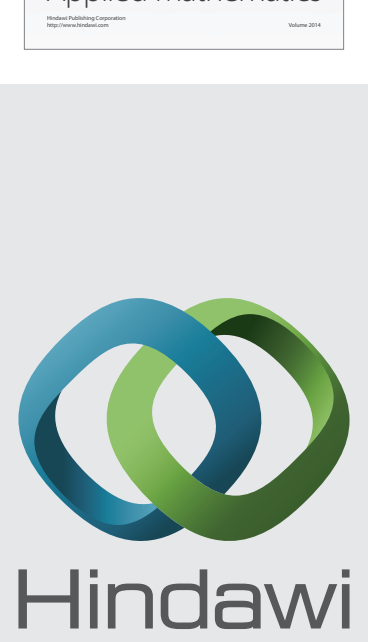

Submit your manuscripts at http://www.hindawi.com
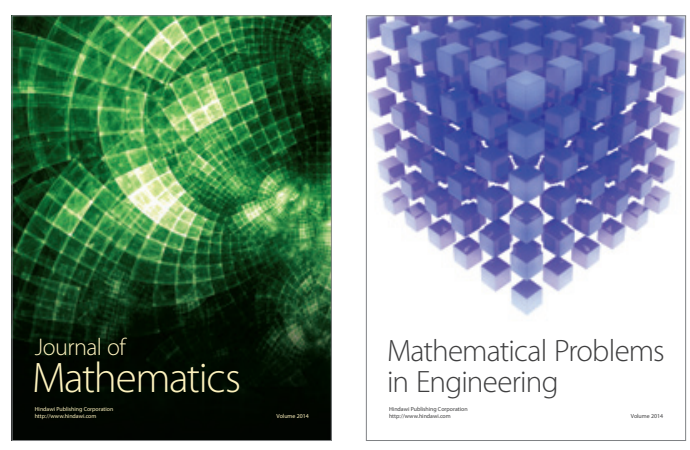

Mathematical Problems in Engineering
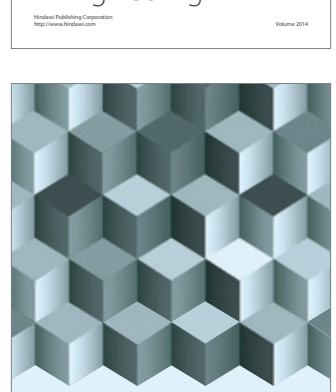

Journal of

Function Spaces
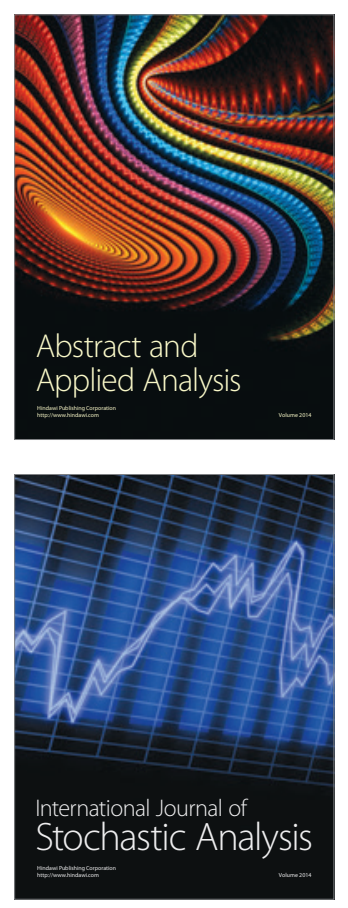

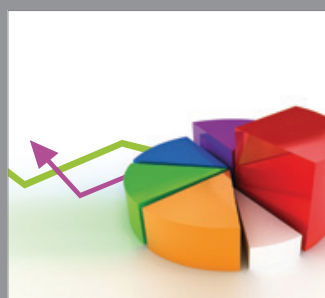

ournal of

Probability and Statistics

Promensencen
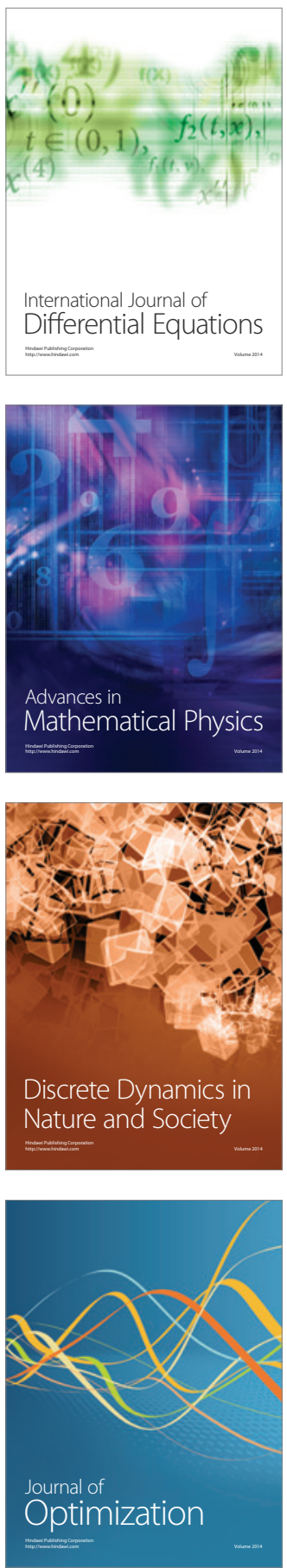